\title{
Influencia de edad y escolaridad en la Percepción de delincuencia y situación económica de las mujeres que habitan Ciudad Obregón, Sonora
}

\section{Influence of age and schooling in the perception of delinquency and economic situation of the women who live in Ciudad Obregon, Sonora}

\author{
OCHOA-JAIME, Blanca Rosa $\uparrow^{*}$, VALDEZ-PINEDA, Dina Ivonne y RUIZ-CORRALES, Missael \\ Instituto Tecnológico de Sonora. Departamento de Ciencias Administrativas. Calle 5 de Febrero 818, Centro, Urb. No. 1, \\ 85000 Cd Obregón, Son.
}

ID $1^{\text {er }}$ Autor: Blanca Rosa, Ochoa-Jaime / ORC ID: 0000-0002-3387-632X, Researcher ID Thomson: S-6886-2018, CVU CONACYT ID: 688073

ID $1{ }^{\text {er }}$ Coautor: Dina Ivonne, Valdez-Pineda / ORC ID: 0000-0001 8660-1787, Researcher ID Thomson: S6886-2018, CVU CONACYT ID: 282771

ID $2^{\text {do }}$ Coautor: Misael, Ruiz-Corrales / ORC ID: 0000-0001 8660-1787, Researcher ID Thomson: S6886-2018, CVU CONACYT ID: 282771

\begin{abstract}
Resumen
El incremento de violencia, inseguridad e impunidad han acrecentado la inconformidad de la sociedad que siente que la delincuencia ha rebasado todos los limites trayendo como efecto una sensación de miedo y angustia, por lo que el objetivo es determinar si influye la edad y el nivel de estudios en la percepción que tienen las mujeres de Ciudad Obregón, Sonora en temas relacionados con Seguridad y Situación Económica. Se toman como base las variables de situación económica y seguridad que componen el Índice de Confianza Ciudadana para medir la percepción de mujeres de Ciudad Obregón en relación a dichas variables. Se elige a las mujeres como sujeto de estudio dado que muchas investigaciones muestran al género femenino con el mayor porcentaje de victimización de la violencia. Con una muestra de 270 mujeres clasificadas por edad y nivel de escolaridad se encuentra que existen diferencias significativas en cuanto a la percepción que tienen ambos grupos, en este caso sin importar edad o nivel de escolaridad la delincuencia es un tema que preocupa a las mujeres, es especial a las mujeres de más de 50 años y a las mujeres con carreras universitarias.
\end{abstract}

Seguridad, Instituciones Gubernamentales,

\begin{abstract}
The increase in violence, insecurity and impunity have increased the dissatisfaction of society that feels that crime has exceeded all limits bringing as effect a sense of fear and anguish, so the objective is to determine if age and level of influence studies on the perception that women in Ciudad Obregon, Sonora have on issues related to Security and Economic Situation. The variables of economic situation and security that compose the Citizen Trust Index are used to measure the perception of women in Ciudad Obregon in relation to these variables. Women are chosen as subject of study given that many investigations show the female gender with the highest percentage of victimization of violence. With a sample of 270 women classified by age and level of schooling, it is found that there are significant differences in the perception of both groups, in this case regardless of age or level of schooling, delinquency is an issue that concerns women, It is special to women over 50 and to women with university careers.
\end{abstract}

Security, Governmental Institutions, Performance Desempeño

Citación: OCHOA-JAIME, Blanca Rosa, VALDEZ-PINEDA, Dina Ivonne y RUIZ-CORRALES, Missael. Influencia de edad y escolaridad en la Percepción de delincuencia y situación económica de las mujeres que habitan Ciudad Obregón, Sonora. Revista de Sociología Contemporánea. 2019. 6-21: 1-8.

\footnotetext{
* Correspondencia del Autor (correo electrónico: blanca.ochoa@itson.edu.mx)

$\dagger$ Investigador contribuyendo como primer autor.
} 


\section{Introducción}

En algunos ámbitos se ha planteado la posibilidad y la necesidad de entender el miedo o la preocupación al crimen, no ya desde una óptica puramente criminológica vinculada al ámbito de la delincuencia, sino como un componente de una temática mayor: la percepción de seguridad que desde una perspectiva de calidad de vida incluye aspectos ambientales, laborales y de participación ciudadana, entre otros (Ospina, 2006).

El miedo a la violencia contra personas independientemente del género nació en el núcleo del malestar ciudadano a mediados de la década de 1960 en los EE.UU. (Stanko, 2000). Los múltiples puntos de contra entre las cuestiones de raza, delincuencia y agitación social formaron la base del miedo a la delincuencia.

La visión general de Hale (1996) sobre el miedo a la delincuencia destaca que se ha debatido sobre la forma de interpretar el miedo a la delincuencia como un indicador de la fragmentación social. A pesar de esto, la creación del concepto de miedo a la delincuencia motivó la limitación de este mismo concepto, puesto que quedaba rodeado y enmarcado por las preocupaciones criminológicas sobre la delincuencia y la mala educación, y ha dejado fuera las implicaciones económicas.

El miedo a la delincuencia se convirtió en problema por méritos propios y muy pronto pasó a ser un problema para las mujeres y para los ancianos, puesto que estos dos grupos sociales ocupan los primeros puestos de la clasificación del miedo. Las encuestas sobre la delincuencia se convirtieron en una herramienta muy popular para redituar el concepto de miedo a la delincuencia en el discurso político de la ciudadanía de las mujeres. El derecho a la seguridad en el espacio público, a andar por la calle tranquila y sin amenazas, se convirtió en uno de los puntos principales de las campañas de las mujeres contra la violencia de género. (Stanko, 1995 y 1997); Madriz, 1997).
Como consecuencia de este estado de alerta, las mujeres restringen las actividades en público a causa de la ansiedad que provoca la posibilidad de que se produzcan actos violentos y aplican más precauciones que los hombres (Gardner, 1995) las rutinas de preocupación, al ser las mujeres más susceptibles a ser víctimas de la delincuencia repercute en muchos casos en su situación económica actual y su situación futura sin importar el rango de edad, profesión o nivel socioeconómico.

En este sentido esta investigación tiene como objetivo principal analizar la percepción de las mujeres en cuanto a la situación económica que provoca la delincuencia en su ciudad, se busca probar si la preocupación acerca de la delincuencia es mayor que la preocupación acerca de la situación económica.

Se tomó como objeto de estudio a las mujeres dado que según los argumentos anteriores son ellas quienes presentan mayores niveles de preocupación en relación a este tema. Para este caso se utilizó la investigación de Ruiz, Ochoa y Plascencia (2017) quienes presentan un indicador de percepción social basado tanto en la delincuencia como en la situación económica, al que denominaron Índice de Confianza Ciudadana (ICC). Se toma esta metodología y las variables que componen dicho índice para probar en dos grupos de mujeres clasificadas por edad y escolaridad, su percepción acerca de dichos componentes.

\section{Desarrollo}

Muchos estudios muestran que en su mayoría, las víctimas de la delincuencia son mujeres, sin importar su edad, estatus social o formación académica, esta situación ha llevado a que formas de justicia privada tiendan a aparecer en condiciones de alto miedo al crimen y, al mismo tiempo, de baja eficacia percibida en el sistema judicial, ya que se ha encontrado que un mayor miedo al delito, una alta insatisfacción con la policía y unas actitudes más punitivas hacia la delincuencia se relacionan entre sí (Bernard, 1991; Cumberland y Zamble, 1992; Ito, 1993).

Entre los efectos sociales del miedo a la victimización está el dejar de lado la preocupación por otras situación pudieran repercutir en la calidad de vida de los habitantes de una ciudad. 
El gasto en seguridad privada o sistemas de seguridad repercuten de manera directa en la estabilidad económica actual y futura de un hogar. Según estos autores, si los habitantes de una comunidad perciben el lugar en el que viven como peligroso por la presencia de sumergidos sociales bandas, prostitución, venta de drogas, pueden ir trasladándose a otras zonas más seguras, lo que trae como consecuencia que sus anteriores residencias se deterioran, bajan los precios de los arriendos y en el lugar acaban residiendo personas con bajos recursos económicos, entre ellos delincuentes, con lo que las tasas de delitos aumentan confirmando lo que temían y percibían los antiguos residentes.

En términos empíricos, los estudios que relacionan la criminalidad y la situación económica local llegan a un cierto consenso en el cual se presentan al menos tres problemas para el análisis de dicho fenómeno: información, medición y percepción. La información es un problema de confiabilidad, debido a que la mayor parte de esta se genera a partir de agencias de seguridad púbica que recogen datos sobre el crimen, pero dichas agencias carecen de credibilidad; por la naturaleza del fenómeno en cuanto a la medición existen sesgos debido a la cantidad de denuncias, las cuales llevan un proceso engorroso, largo y burocrático, por lo cual existe falta de credibilidad en los datos, temor y ambigüedad, las encuestas de victimización son costosas y poco efectivos por el hecho de basarse en la memoria y tener una metodología de recolección basada en la conveniencia, es decir, basada en quienes desean contestar; en la medición de la delincuencia o criminalidad pueden emplearse tasas reales o tasas percepcionales, lo cual lleva a analizar el tercer punto, las tasas reales miden la criminalidad, y las percepcionales miden el temor al crimen, lo cual son dos indicadores diferentes, la percepción impacta en decisiones de ubicación, en los gastos de los hogares, en las decisiones de voto entre otras (Kliksberg, 2002). Existen algunos estudios que muestran la relación entre la criminalidad o inseguridad y la situación económica de una región, por ejemplo Lozano (2011) llega a la conclusión de que existe una fuerte interacción negativa entre la delincuencia y el crecimiento económico, lo anterior a partir de un análisis factorial de componentes principales, que les permitió calcular un índice de seguridad pública y un índice de seguridad privada.
Diversos estudios empíricos muestran la relación entre la seguridad y la situación económica de ciertas regiones; resultados que indican que el crecimiento del Producto Interno Bruto per cápita real en una entidad federativa presenta relaciones unidireccionales positivas con el crecimiento de otras entidades cercanas, relaciones negativas con entidades cuyos índices de crecimiento delictivo es considerablemente, y relaciones positivas directas con los índices de criminalidad de la misma entidad en periodos anteriores, los resultados anteriores obtenidos a través de un análisis auto regresivo (Pan et $a$; 2012).

Utilizando también un modelo auto regresivo, Detotto y Otranto (2010) prueban que existe un impacto significativo en un sentido negativo y proporcional del crimen hacia el PIB real, y dicho impacto presenta una magnitud que depende del desempeño de las economías, siendo los momentos de crisis o recesión de dichas economías, el periodo en el cual la magnitud de los efectos negativos se potencializan, pues ante un incremento de una unidad porcentual en la tasa de homicidios, existe un incremento en el mismo sentido en el costo económico asumido por las economías, dicho costo es cinco por ciento más cuando se trata de una economía en proceso de recesión, que cuando se trata de una economía en proceso de expansión.

Por su parte Ruiz, Ochoa y Plascencia, (2017) presentan argumentos y prueban la relación de la delincuencia con la situación económica a través de un índice, que se basa en la percepción de los habitantes de una ciudad, en este sentido dicha percepción sirve de base para analizar la medida en la cual la percepción de las mujeres varía en función tanto de su edad como de su escolaridad, para lo cual se desarrolló una investigación que de acuerdo con Hernández, Fernández, y Baptista (2010) posee un alcance descriptivo y cuantitativo elaborado con un diseño no experimental. En base al tiempo en el cual fue aplicada la investigación, esta es de corte transversal, debido a que la información de las personas encuestadas, fue recabada durante el mismo periodo de tiempo. 
Las variables que componen el Índice de Confianza Ciudadana (ICC) se determinaron en base a Ruiz, Ochoa y Plascencia (2017), (ver tabla 1, tabla 2, tabla 3), la clasificación de los rangos de edades y nivel escolaridad de las mujeres encuestadas se basó en la clasificación que realiza el Instituto Nacional de Estadística y Geografía (INEGI) a través de la "Encuesta Nacional de Seguridad Pública Urbana” ENSU, (2018); que genera estimaciones sobre la percepción de la población sobre la seguridad pública y en la "Encuesta Nacional Sobre Confianza del Consumidor” ENCO, (2018).

\begin{tabular}{|c|c|c|c|}
\hline Dimensión & Variables & Pregunta & $\begin{array}{l}\text { Opciones } \\
\text { de } \\
\text { respuesta }\end{array}$ \\
\hline Seguridad & Delincuencia & $\begin{array}{l}\text { En términos de } \\
\text { delincuencia, } \\
\text { ¿Considera que } \\
\text { vivir en su Ciudad } \\
\text { es?: }\end{array}$ & $\begin{array}{l}\text { 1.Seguro } \\
\text { 2. Inseguro } \\
\text { 3. No } \\
\text { sabe/No } \\
\text { responde }\end{array}$ \\
\hline \multirow[t]{5}{*}{$\begin{array}{l}\text { Situación } \\
\text { Económica }\end{array}$} & $\begin{array}{l}\text { Situación } \\
\text { Económica } \\
\text { actual del } \\
\text { hogar }\end{array}$ & $\begin{array}{l}\text { Comparada con la } \\
\text { situación } \\
\text { económica que los } \\
\text { miembros de este } \\
\text { hogar tenían hace } \\
12 \text { meses, ¿Cómo } \\
\text { cree que es su } \\
\text { situación este } \\
\text { momento? }\end{array}$ & $\begin{array}{l}\text { 1. Mucho } \\
\text { mejor. } \\
\text { 2. Mejor } \\
\text { 3. Igual } \\
\text { 4. Peor } \\
\text { 5. Mucho } \\
\text { peor } \\
\text { 6. No sabe. }\end{array}$ \\
\hline & $\begin{array}{l}\text { Situación } \\
\text { económica } \\
\text { futura del } \\
\text { hogar. }\end{array}$ & $\begin{array}{l}\text { ¿Cómo considera } \\
\text { usted que será la } \\
\text { situación } \\
\text { económica de los } \\
\text { miembros de éste } \\
\text { hogar dentro de } 12 \\
\text { meses, respecto a la } \\
\text { actual? }\end{array}$ & $\begin{array}{l}\text { 1. Mucho } \\
\text { mejor. } \\
\text { 2. Mejor } \\
\text { 3. Igual } \\
\text { 4. Peor } \\
\text { 5. Mucho } \\
\text { peor } \\
\text { 6. No sabe. }\end{array}$ \\
\hline & $\begin{array}{l}\text { Situación } \\
\text { Económica } \\
\text { actual de la } \\
\text { Ciudad. }\end{array}$ & $\begin{array}{l}\text { ¿Cómo considera la } \\
\text { situación } \\
\text { económica de la } \\
\text { Ciudad hoy día } \\
\text { comparada con la } \\
\text { de hace } 12 \text { meses? }\end{array}$ & $\begin{array}{l}\text { 1. Mucho } \\
\text { mejor. } \\
\text { 2. Mejor } \\
\text { 3. Igual } \\
\text { 4. Peor } \\
\text { 5. Mucho } \\
\text { peor } \\
\text { 6. No sabe. }\end{array}$ \\
\hline & $\begin{array}{l}\text { Situación } \\
\text { económica } \\
\text { futura de la } \\
\text { Ciudad }\end{array}$ & $\begin{array}{l}\text { ¿Cómo considera } \\
\text { usted que será la } \\
\text { condición } \\
\text { económica de su } \\
\text { Ciudad dentro de } 12 \\
\text { meses respecto de la } \\
\text { actual situación? }\end{array}$ & $\begin{array}{l}\text { 1. Mucho } \\
\text { mejor. } \\
\text { 2. Mejor } \\
\text { 3. Igual } \\
\text { 4. Peor } \\
\text { 5. Mucho } \\
\text { peor } \\
\text { 6. No sabe. }\end{array}$ \\
\hline & $\begin{array}{l}\text { Posibilidad } \\
\text { actual de } \\
\text { realizar } \\
\text { compras. }\end{array}$ & $\begin{array}{l}\text { Comparando la } \\
\text { situación } \\
\text { económica actual } \\
\text { con la de hace un } \\
\text { año. ¿Cómo } \\
\text { considera en el } \\
\text { momento actual las } \\
\text { posibilidades de } \\
\text { que usted o alguno } \\
\text { de los integrantes de } \\
\text { este hogar realice } \\
\text { compras tales como } \\
\text { muebles, televisor, } \\
\text { lavadoras, otros } \\
\text { aparatos } \\
\text { electrodomésticos, } \\
\text { etc.? }\end{array}$ & $\begin{array}{l}\text { 1.Mayores } \\
\text { 2.Iguales } \\
\text { 3.Menores } \\
\text { 4. No sabe. }\end{array}$ \\
\hline
\end{tabular}

Tabla 1 Descripción de las variables Fuente: Ruiz, Ochoa y Plascencia (2017)
Para calcular el ICC se tomaron 7 preguntas, 1 para medir la dimensión de seguridad, 5 para medir la situación económica y 1 más como ponderador para determinar el peso entre situación económica y seguridad. Para determinar la percepción en relación en la dimensión de seguridad, se utilizó la variable y la pregunta descrita en la tabla 1, cuyas posibles respuestas y ponderaciones se presentan en la tabla 2.

\begin{tabular}{|l|r|}
\hline \multicolumn{1}{|c|}{ Opciones de respuesta } & \multicolumn{2}{c|}{ Ponderador } \\
\hline Seguro & 1.00 \\
\hline Inseguro & 0.00 \\
\hline No sabe & NA \\
\hline
\end{tabular}

Tabla 2 Ponderador dimensión seguridad Fuente: Ruiz, Ochoa y Plascencia (2017)

A partir del ponderador se obtiene un índice para la dimensión seguridad. Para el caso de la dimensión situación económica, el índice resulta de promediar los índices de las 5 variables que miden dicha dimensión. A su vez, cada componente se calcula como el promedio ponderado de respuestas expandidas de cada una de las preguntas referentes a cada una de las variables que miden la dimensión, y cuya descripción se presenta en la tabla 1 .

En cada una de las cuatro primeras preguntas los entrevistados tienen cinco opciones de respuesta: mucho mejor, mejor, igual, peor y mucho peor. En la quinta pregunta las opciones son: mayores, iguales y menores. Los ponderadores utilizados para cada opción de respuesta se presentan en la tabla 3 .

\begin{tabular}{|c|c|c|}
\hline \multirow[b]{2}{*}{$\begin{array}{l}\text { Opciones de } \\
\text { respuesta }\end{array}$} & \multicolumn{2}{|c|}{ Ponderador } \\
\hline & $\begin{array}{c}\text { Primeras } 4 \\
\text { preguntas }\end{array}$ & $\begin{array}{c}\text { Ultima } \\
\text { pregunta }\end{array}$ \\
\hline Mucho mejores & 1.00 & \\
\hline Mejores (mayores) & 0.75 & 1.00 \\
\hline Igual (iguales) & 0.50 & 0.50 \\
\hline Peor (menores) & 0.25 & 0.00 \\
\hline Mucho peor & 0.00 & \\
\hline
\end{tabular}

Tabla 3 Ponderador dimensión situación económica Fuente: Ruiz, Ochoa y Plascencia, (2017)

Para obtener el ICC debe sumarse el resultado de multiplicar el Índice de seguridad, y el índice de situación económica por el ponderador de preocupación, el cual se determina a través de preguntar a los encuestados que les preocupa más, si la situación económica o la inseguridad. 
Se estudió a los habitantes Ciudad Obregón Sonora, en México, considerando una muestra no probabilística y a conveniencia de 200 personas, las cuales decidieron contestar la encuesta y cumplían con las características de ser mayores de edad y habitar en la Ciudad objeto de estudio. De los 200 encuestados se tomó solo en cuenta aquellos que fueran mujeres, por lo cual la muestra para este estudio en específico es de 114 mujeres.

Se utilizó el instrumento propuesto por Ruiz, Ochoa y Plascencia (2017) para medir la percepción acerca de la seguridad y la situación económica de la ciudad, dicho instrumento consta de 80 reactivos, las cuales miden aspectos generales del encuestador y los encuestados, como dirección, correo electrónico, sexo, edad, ocupación, entre otras, y aspectos de su percepción en relación a la situación económica y seguridad de su ciudad.

Para el contraste de cada variable que compone el ICC y los grupos que componen tanto la escolaridad como la edad de las mujeres se presentan de la siguiente manera:

$\begin{array}{ll}\text { Escolaridad } & \text { Rango de Edad } \\ \text { Básica } & 18-25 \\ \text { Media Superior } & 26-38 \\ \text { Licenciatura } & 39-50 \\ \text { Posgrado } & \text { Más de } 30\end{array}$

Para determinar si existe diferencia en cuanto al comportamiento de las variables que componen el ICC a la escolaridad y la edad de las mujeres, se utiliza el análisis de la varianza de un factor (ANOVA), el cual es útil para comparar varios grupos en una variable cuantitativa. Se refiere, por lo tanto, de una generalización de la prueba $\mathrm{T}$ de student, para dos tipos de muestras independientes, al caso de diseño con más de dos muestras. La variable que se encarga de definir los grupos que se desea comparar es llamada independiente. La variable cuantitativa en la que se desea comparar los grupos se le llama variable dependiente. La hipótesis que se evalúa con el ANOVA de un factor se saca por la igualdad de media la cual existe entre la variable dependiente y en todos los factores que componen la variable independiente. Si existe similitud de medias quiere decir que cada factor se comporta de un igual manera y por resultado ambas variables son independientes una de la otra, por lo cual no existe ninguna relación.
La estrategia utilizada para evaluar la hipótesis de igualdad de medias consiste en la obtención de un estadístico, llamado F, el cual refleja el grado de similitudes existentes entre las medias que se están comparando. Si las poblacionales muestreadas son normales y sus varianzas son iguales, el estadístico $F$ se distribuye según el modelo de probabilidad $\mathrm{F}$ de Fisher-Snedecor. Si se supone cierta la hipótesis de igualdad de medias, se puede conocer en todo momento la probabilidad de obtener un valor como el obtenido o mayor (Pardo y San Martin, 1998). Se realizan dos pruebas; en la primera se realiza el procedimiento ANOVA de un factor para medir las variaciones de las variables del ICC en relación a los grupos que componen la edad de las mujeres. La segunda prueba repite en procedimiento anterior pero ahora en función de la escolaridad; es importante mencionar que ambas pruebas se realizan con un nivel de confianza del $90 \%$.

\section{Resultados}

A continuación, se presentan una serie de resultados que permiten contestar la pregunta de investigación y cumplir con los objetivos planteados. Para el primer análisis que muestra la relación entre las 6 variables que componen el ICC y el rango de edad de las mujeres, el cálculo del ANOVA de un factor presentado permite comparar las medias y muestra que el estadístico $\mathrm{F}$ a un nivel de confianza del $95 \%$ permite rechazar la hipótesis de igualdad de medias de las variables: Delincuencia, situación económica actual del hogar y situación económica futura de la ciudad; pero no permite rechazar la hipótesis de igualdad de medias en las otras tres variables, es decir, la percepción de los mujeres varía en función de 3 de las 6 variables que componen el ICC dependiendo del rango de edad de dichas mujeres, las otras 3 variables no presentan alteraciones. Para dar soporte a los argumentos de la diferencia de media encontrada en el ANOVA de un factor se realizó un análisis post-hoc que mide la distancia de las diferencias de grupos basados en Bonferroni, dichos resultados se presentan en la tabla 4. 


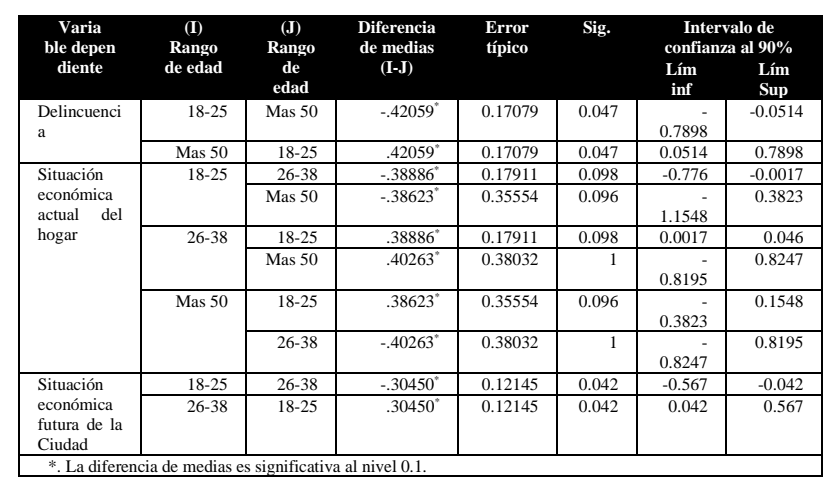

Tabla 4 Comparaciones múltiples (Edad) Fuente: elaboración propia

Es importante denotar que en las tablas de comparaciones múltiples tanto de edad como de escolaridad solo se toma en cuenta aquellos grupos que presentan diferencias de medias significativas, dejando de lado aquellas cuya diferencia no es significativa para este análisis. Las comparaciones múltiples permiten medir el nivel de valoración por rango de edad de las mujeres en relación a las 3 variables que presentaron diferencias en la percepción, en este caso se puede observar que en lo referido a delincuencia, la diferencia en la percepción se da entre las mujeres con un rango de edad de 18-25 y las mayores de 50 años.

Son estas últimas quienes muestran un sentido de preocupación mayor que las mujeres más jóvenes; para el resto de rango de edades no hay diferencia significativa, lo anterior no significa que no se preocupen por la delincuencia, solo que esa preocupación no es estadísticamente significativa comparada con los rangos de edad de 18-25 y más de 50.

Para el caso de la variable denominada situación económica actual del hogar, de los 4 grupos de edad, tres de ellos presentan diferencias de medias en cuanto a la percepción de esta variable, es este caso en específico tanto las mujeres de 26-38 como las de más de 50 se preocupan más que de las de 18-25 en relación a la situación económica actual de sus hogares, es importante denotar que en relación a este tema, las más preocupadas son las mujeres de 26-38 años, lo anterior podría estar explicado por el hecho de que es el rango de edad laboral, en este sentido las mujeres de esas edades están o buscando un empleo o consolidándose como profesionistas, lo cual podría explicar su mayor preocupación por la situación económica actual de su hogar.
Por último, y en relación a la variable, situación económica futura de la ciudad solo existe diferencia de media en dos de los cuatro grupos de este análisis, en este caso son las mujeres de un rango de edad de 26-38 años quienes muestran niveles de preocupación mayor en relación al incierto futuro económico de Ciudad Obregón, comparada con las mujeres de un rango de edad de entre los 18 y 25 años. Se repitió el procedimiento del ANOVA de un factor pero en este caso teniendo como elemento de comparación la escolaridad de las mujeres, la tabla número 5 se muestran los resultados de las comparaciones múltiples.

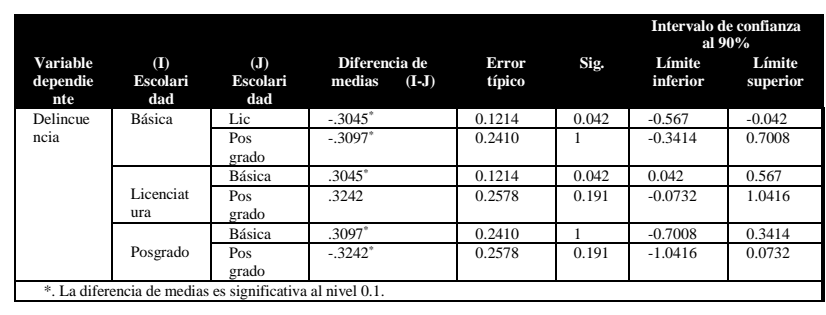

Tabla 5 Comparaciones múltiples (Escolaridad)

Fuente: elaboración propia

En cuanto a la escolaridad, es importante notar, que la tabla anterior deja fuera de análisis a aquellas mujeres cuyo máximo grado de estudios es el nivel medio superior, no porque no sea relevante, sino que para el caso específico de este análisis no presenta diferencia de medias significativas en cuanto a la percepción de las variables que componen el ICC.

Observando la tabla 4, se pone en evidencia que las mujeres de la muestra presentan diferencias de percepción solo en la variable delincuencia, es evidente que la preocupación en este rubro es superior que en el resto de las variables, para este caso tanto las mujeres que tienen una licenciatura, como las que tienen un posgrado presentan un nivel de preocupación mayor en cuanto a la delincuencia que aquellas mujeres cuyos estudio máximos están en el nivel básico, sin embargo la preocupación es mayor por parte de las mujeres con posgrado que las mujeres con una licenciatura, lo anterior podría tener sus orígenes en el hecho de que un posgrado generalmente implica mayores ingresos, por lo cual se es más probable ser objeto de la delincuencia. 


\section{Conclusiones}

Los resultados obtenidos en base a las pruebas realizadas permiten cumplir con el objetivo establecido para este estudio. Basado en eso, se obtuvieron una serie de conclusiones.

- A un nivel de confianza del $90 \%$ existe diferencia de medias en tres de los seis variables que componen el Índice de Confianza Ciudadana que componen en función de la edad de las mujeres.

- La variación en la percepción de las mujeres acerca de las variables que componen el ICC en función de la edad presenta variaciones en las variables delincuencia, situación económica actual del hogar, situación económica futura de la ciudad.

- Para el caso del análisis por grupos de edad, y en relación a la delincuencia son las mujeres mayores de 50 años quienes muestran un nivel de preocupación mayor que las de 1825 , los otros grupos no muestran diferencias en cuanto a esta variable.

- Tanto las mujeres de 26-38 como las de más de 50 se preocupan más que de las de 18-25 en relación a la situación económica actual de sus hogares, es importante denotar que en relación a este tema, las más preocupadas son las mujeres de 26-38 años. Y para el caso de la situación económica futura de la ciudad son las mujeres de 26 a 38 años quienes muestran un nivel de preocupación superior que el resto de los grupos de edades.

- Para el caso del análisis por escolaridad, de las 6 variables que componen el ICC solo existe diferencia en cuanto opiniones o percepciones en la variable delincuencia, esta variable se repite en cuanto al grado de preocupación tanto por edad como por escolaridad, en este caso y sustentado en el hecho de que las personas con un posgrado tienden a tener mayores ingresos, son las mujeres con este nivel de escolaridad quienes presentan mayor grado de preocupación que el resto de las mujeres con niveles escolares inferiores.

- Es importante mencionar que al analizar en ambos grupos (Edad y escolaridad) la variable delincuencia es la que muestra mayor grado de preocupación entre las mujeres.
De manera general y en concordancia con Ruiz, Ochoa y Plascencia (2017) tanto la situación económica como la delincuencia están relacionados, y la percepción de estos elementos puede variar en función de distintos factores. En este caso al ser de manera probada las mujeres quienes son más susceptibles a la delincuencia, su preocupación por este tema es palpable, sin importar el rango de edad o su nivel de escolaridad la delincuencia presenta un alto grado de preocupación en este grupo de la sociedad. Para el caso específico de Ciudad Obregón, Sonora, las mujeres en un rango de edad de más de 50 años y que tienen un posgrado son las que más importancia le dan o mayor grado de preocupación presentan en cuanto a la delincuencia.

\section{Referencias}

Bernard, Y. (1991). North American and European research on fear of crime. Applied Psychology: An International Review/Psychlogie Appliqué: revue internationale, 41; 65-77.

Cumberland, J. \& Zamble, E. (1992). General and Specific Measures of Attitudes Toward Early Release of Criminal Offenders. Canadian Journal of Behavioural Science, 24; (4) 442-455.

Detotto, C. y Otranto, D. (2010) Does crime Affect Economic Growth?. Kyklos, International Review for Social Science, Estados Unidos, Blackwell publishing. 63; (3). 330-345.

Encuesta Nacional sobre Confianza del Consumidor. (2017) Instituto Nacional de Estadística y Geografía (INEGI) [en línea] http://www.beta.inegi.org.mx/proyectos/enchog ares/regulares/enco/ [Consulta: 17-12-2018].

Encuesta Nacional de Seguridad Pública Urbana. (2017), Instituto Nacional de Estadística y Geografía (INEGI) [en línea] http://www.beta.inegi.org.mx/proyectos/enchog ares/regulares/ensu/ [Consulta: 17-12-2018].

Gardner, C. (1995) Passing by: Gender and Public Harassment. Berkeley, California: University of California Press.

Hale, C. (1996) Fear of Crime: A review of the literature. International Review of Victimology, 4, p. $79-150$

OCHOA-JAIME, Blanca Rosa, VALDEZ-PINEDA, Dina Ivonne y RUIZ-CORRALES, Missael. Influencia de edad y escolaridad en la Percepción de delincuencia y situación económica de las mujeres que habitan Ciudad Obregón, Sonora. Revista de Sociología Contemporánea. 2019 
Ito, R. (1993). Research on the fear of crime: perceptions and realities of crime in Japan. Crime \& Delinquency, 139; (3), 385-392.

Kliksberg, B. (2002) El crecimiento de la criminalidad en América Latina: un tema urgente", Multiciencias, Venezuela. 2; (2) 8591.

Lozano, C. (2011) Estudio comparativo de la cárcel en España y México en la actualidad. Estudios Sociales. 20; (40) 98-124.

Madriz, E. (1997a) Nothing Bad Happens to Good Girls. Berkeley, California: University of California Press.

Ospina, J. (2006). La percepción de seguridad en Bogotá. $1^{\text {o }}$ Seminario Internacional de Percepción de Seguridad Ciudadana. Bogotá, 18 y 19 de Abril. Peña, J.A. (2005). Miedo al delito en Bog.

Pan, M. Widner, B. y Enomoto, C. (2012) Growth and crime in contiguous States of México, Review of Urban and Regional Development Studies, New Mexico, New Mexico State University, vol. XXIV, núm. 1/2, pp. 51-64.

Ruiz, M. Ochoa, B. Plascencia, I. (2017) Indicadores de confianza ciudadana como medida del desempeño y efectividad de las instituciones gubernamentales, un caso mexicano, Colombia, Revista Criterio Libre, vol. 15, núm. 27.

Stanko, E. (1995) Women, crime and fear. Annals of the American Academy of Political and Social Science. 539; 46-58.

Stanko, E. (1997) Homophobic violence and the self "at risk": interrogating the boundaries. Social and Legal Studies. 6; (4) 513-532.

Stanko, E. (2000) Safety Talk: Conceptualising Women's risk Assessment as a "Technology of the Soul". Theoretical Criminology. 1; (4)47999. 